\title{
AN AVERAGING PROPERTY OF THE RANGE OF A VECTOR MEASURE
}

\author{
BY J. DIESTEL ${ }^{1}$ AND C. J. SEIFERT
}

Communicated by R. G. Bartle, April 19, 1976

Our discussion centers around the striking properties displayed by the range of a vector-valued measure. Let $\Sigma$ be a $\sigma$-field of sets, $X$ be a Banach space and $F: \Sigma \rightarrow X$ be a countably additive map (a vector measure). Bartle, Dunford and Schwartz [3] showed that $F(\Sigma)$ is relatively weakly compact; Liapounov [13] (see also Lindenstrauss [14]) showed that if $X$ is finite dimensional then $F(\Sigma)$ is compact and, if $F$ has no atoms, convex. Some additional peculiarities: Each extreme point of the closed convex hull of $F(\Sigma), \overline{\operatorname{co}}(F(\Sigma))$, lies in $F(\Sigma)$ [12]. Each extreme point of the closed convex hull of $F(\Sigma)$ is a denting point of $\overline{\operatorname{co}}(F(\Sigma))$ [1]. The exposed points of $\overline{\operatorname{co}}(F(\Sigma))$ are strongly exposed [1] and a point $x \in \overline{\operatorname{co}}(F(\Sigma))$ is exposed by $x^{*} \in X^{*}$ (the dual of $X$ ) if and only if $F$ is $\left|x^{*} F\right|$-continuous. While any two dimensional unit ball is the range of a vector measure, the unit ball of an $l_{p}^{3}(1 \leqslant p<2)$ is not ([4], [7]). Kluvanek [10] has noted that as a consequence of a classical theorem of Banach [8] the unit ball of $l_{2}$ is the range of a vector measure; he [11] has also obtained a characterization of the range of vector measures. The closed unit ball of $L_{p}$ (or $l_{p}$ ) for $1<p<2$ is not the range of a vector measure. Since this last assertion seems not to be easily deducible from Kluvanek's characterization, a few remarks on its proof are in order: Note that if the ball of $X$ is the range of a vector measure $F$ then $X$ is the quotient via integration of the Banach space $B(\Sigma)$ of bounded $\Sigma$ measurable functions-a $C(K)$ space. If $X$ is also a subspace of some $L_{1}$ space then Grothendieck's inequality [15] implies $X$ is isomorphic to a Hilbert space. Since $L_{p}[0,1]$ is isomorphic to a subspace of $L_{1}[0,1]$ by [5] but is not isomorphic to any Hilbert space [2] our original assertion follows.

Our main result is built upon the beautiful paper of Szlenk [16] and using his methods we have the

THEOREM. Every sequence in the range of a vector measure has a subsequence whose arithmetic means are norm convergent.

OUTLINE OF PROOF. Key to the proof is the fact proved by Bartle, Dunford and Schwartz [3] that there exists a probability measure $\mu$ on $\Sigma$ with the same null sets as $F$. Look at a sequence $\left(F\left(E_{n}\right)\right)$ chosen from the range of the vector

AMS (MOS) subject classifications (1970). Primary 28A45.

${ }^{1}$ This author's research was supported in part by NSF MPS 08050. 
measure $F$; there exists a sequence $\left(x_{m}^{*}\right)$ in $X^{*}$ such that for each $x$ in the closed linear span of $\left\{F\left(E_{n}\right)\right\},\|x\|=\sup _{m}\left\{\left|x_{m}^{*}(x)\right|\right\}$, where $\left\|x_{m}^{*}\right\|=1$ for all $m$. For each $n$, let $F_{n}: \Sigma \rightarrow X$ be defined by $F_{n}(A)=F\left(E_{n} \cap A\right)$. Each $F_{n}$ is an $X$ valued countably additive $\mu$-continuous measure. Moreover, the family $\left\{x_{m}^{*} F_{n}\right.$ : $m, n=1,2, \ldots\}$ is uniformly absolutely continuous with respect to $\mu$ and so by the Radon-Nikodym Theorem this family can be viewed as a uniformly integrable bounded subset of $L_{1}(\mu)$. Given any increasing sequence $\left(n_{k}\right)$ of positive integers, it is easily established that

$$
\left\|\frac{1}{p} \sum_{k=1}^{p} F\left(E_{n_{k}}\right)-\frac{1}{q} \sum_{k=1}^{q} F\left(E_{n_{k}}\right)\right\| \leqslant \sup _{m}\left\|\frac{1}{p} \sum_{k=1}^{p} x_{m}^{*} F_{n_{k}}-\frac{1}{q} \sum_{k=1}^{q} x_{m}^{*} F_{n_{k}}\right\|_{L_{1}(\mu)} .
$$

From this it is easy to mimic the closing steps (Lemma 2 and Theorem) of Szlenk [16] to obtain the desired conclusion.

CoRollary. A weakly compact order interval in a Banach lattice is the range of a vector measure, consequently it has the Banach-Saks property.

Proof. If $\langle 0, x\rangle$ is an order interval, then the gauge \|\|$_{x}$ of $\langle-x, x\rangle$ is a lattice norm on the linear span $L_{x}$ of $\langle-x, x\rangle$. The completion $C$ of $\left(L_{x},\|\|_{x}\right)$ is an $M$-space with unit and so is a $C(K)$-space by Kakutani's representation theorem [9]. If $\langle 0, x\rangle$ is weakly compact, the canonical inclusion of $\left(L_{x},\|\|_{x}\right)$ into $X$ extends to a weakly compact linear operator from $C$ to $X$ which takes the nonnegative members of the unit ball of $C$ onto $\langle 0, x\rangle$. It is a well-known fact that $\langle 0, x\rangle$ is the closed convex hull of the range of the representing vector measure for this extension of the inclusion map (Bartle, Dunford and Schwartz [3]). We now apply the observation of Kluvanek and Knowles [12, Chapter 5, §5] to complete the proof.

\section{REFERENCES}

1. R. Anantharaman, On exposed points of the range of a vector measure, Vector and Operator Valued Measures and Applications (Proc. Sympos., Snowbird Resort, Alta, Utah, 1972), Academic Press, New York, 1973, pp. 7-22. MR 48 \#11436.

2. S. Banach, Théorie des operationes linéaires, Warzawa, 1932.

3. R. G. Bartle, N. Dunford and J. T. Schwartz, Weak compactness and vector measures, Canad. J. Math. 7 (1955), 289-305. MR 16, 1123.

4. E. D. Bolker, $A$ class of convex bodies, Trans. Amer. Math. Soc. 145 (1969), 323345. MR 41 \#921.

5. J. Bretagnolle, D. Dacunha-Castelle and J. L. Krivine, Lois stables et espaces $L^{p}$, Ann. Inst. Poincaré Sect. B (N.S.) 2 (1965/66), 231-259. MR 34 \#3605.

6. J. Diestel, Geometry of Banach spaces: Selected topics, Lecture Notes in Math., no. 485, Springer-Verlag, 1975.

7. L. Dor, Potentials and isometric embeddings in $L_{1}$ (preprint).

8. S. Kaczmarz and H. Steinhaus, Theorie der Orthogonalreihen, Warzawa, 1935.

9. S. Kakutani, Concrete representation of abstract $(M)$-spaces. ( $A$ characterization of the space of continuous functions), Ann. of Math. (2) 42 (1941), 994-1024. MR 3, 205. 
10. I. Kluvanek, $A$ measure whose range contains a ball in $l_{2}$, Notices Amer. Math. Soc. 21 (1974), A-539. Abstract 74T-B206.

11. - The closed convex hull of the range of a vector measure (preprint).

12. I. Kluvanek and G. Knowles, Vector measures and optimal controls (manuscript).

13. A. Liapounoff, Sur les fonctions-vecteurs complètement additives, Izv. Akad. Nauk SSSR Ser. Mat. 4 (1940), 465-478. MR 2, 315.

14. J. Lindenstrauss, $A$ short proof of Liapounoff's convexity theorem, J. Math. Mech. 15 (1966), 971-972. MR 34 \#7754.

15. J. Lindenstrauss and A. PeXczyński, Absolutely summing operators in $L_{p^{-s p a c e s}}$ and their applications, Studia Math. 29 (1968), 275-326. MR 37 \#6743.

16. W. Szlenk, Sur les suites faiblement convergentes dans l'espace L, Studia Math. 25 (1965), 337-341. MR 34 \#1833.

DEPARTMENT OF MATHEMATICS, KENT STATE UNIVERSITY, KENT, OHIO 44242 